\title{
Frequência de ocorrência de cavidade anoftálmica na região centro-oeste paulista e características dos portadores
}

\author{
Frequency of occurrence of anophthalmic socket in the Middle West region of the state of São Paulo \\ and the carriers characteristics
}

Roberta Lilian Fernandes de Sousa ${ }^{1}$, André Ricardo Carvalho Marçon² ${ }^{2}$ Carlos Roberto Padovani³ ${ }^{3}$ Silvana Artioli Schellini ${ }^{4}$

\section{RESUMO}

Objetivo: Observar a frequência de ocorrência de casos de cavidade anoftálmica na região centro-oeste paulista e descrever o perfil demográfico dos portadores em estudo populacional.

Métodos: Estudo transversal, de caráter observacional e de amostragem aleatória, realizado em 12 cidades da região centro-oeste do estado de São Paulo, para as quais o centro de referência é a cidade de Botucatu. Os participantes foram determinados por sorteio que levou em conta o local de moradia, tendo sido estabelecida uma amostra de 11.453 indivíduos. Todos os exames foram feitos utilizando uma Unidade Oftalmológica Móvel. O protocolo da pesquisa consistiu de dados demográficos e exame oftalmológico completo (anamnese, antecedentes oculares e sistêmicos, antecedentes familiares, avaliação da acuidade visual com e sem correção, tonometria, biomicroscopia, fundoscopia e exame refracional). Todos os dados obtidos foram transferidos para tabela Excel e submetidos à análise descritiva e apresentados como frequência de ocorrência.

Resultados: A frequência de ocorrência de cavidade anoftálmica na região centro-oeste paulista foi de 0,96\% . Dentre os sujeitos examinados, foram encontrados 11 casos de cavidade anoftálmica, com acometimento de 0,7\%, no sexo feminino e 1,3\%, do sexo masculino. Dentre as causas de cavidade anoftálmica foram encontradas glaucoma (olho cego doloroso), microftalmia, trauma e endoftalmite. Os indivíduos eram, em sua maioria, de idade superior ou igual a 40 anos, tendo sido encontrado apenas um caso com idade abaixo de 19 anos.

Conclusão: A cavidade anoftálmica ocorreu em 0,96\% dos habitantes da região centro-oeste paulista, acometendo mais frequentemente os homens e com grande variação de idade de acometimento.

Descritores: Procedimentos cirúrgicos oftalmológicos; Cavidade anoftálmica; Epidemiologia; Estudos transversais; Técnicas de diagnóstico oftalmológico; Vigilância da população

\begin{abstract}
Purpose: To observe the frequency of the occurrence of the anophthalmic socket in the Middle West region of the state of São Paulo and to describe the demographic profile of the carriers in a population-based data.

Methods: A cross-sectional study involve a random sampling carried out in twelve cities of the Middle West region of the state of São Paulo, for which the reference center is the city of Botucatu was done. The participators were chosen by assortment which considered the houses of these people. It was established a sampling with 11,453 people. All the exams were realized using a Mobile Ophthalmologic Unit. The research protocolincluded the demographic data and the complete ophthalmologic exam (anamnesis, ocular and systemic antecedents, familiar antecedents, visual acuity with and without correction, tonometry, biomicroscopy, fundoscopy, and refraction exam). All the data were transferred to an Excel spreadsheet and submitted to a descriptive analysis and were presented by the frequency of the occurrence.

Results: Ophthalmic socket frequency in the Middle West region of the state of São Paulo was $0.96 \%$. We found 11 cases of anophthalmic socket, with involvement of $0.7 \%$ in females and $1.3 \%$, male. The most common causes of anophthalmic socket were glaucoma (blind painful eye), microphthalmia, trauma, and endophthalmitis. The majority of the people were 40 years old or more, and we found just one person younger than 19 years old.
\end{abstract}

Conclusion: The anophthalmic socket occurred in 0.96\% of the habitants of the Middle West region of São Paulo State, occurring mainly in male and with large variety of the affected ages.

Keywords: Ophthalmologic surgical procedures; Anophthalmic socket; Epidemiology; Cross-sectional studies; Population surveillance

\section{INTRODUÇÃO}

Órbita anoftálmica ou cavidade anoftálmica é o nome dado à órbita que se apresenta sem o olho, ausente devido a causa congênita ou após remoção por meio de evisceração ou enucleação. A remoção do olho é a indicação cirúrgica do estágio final de várias doenças oculares, tais como glaucoma absoluto, phthisis bulbi, panuveítes; trauma com perda substancial de conteúdo e nos quais a reconstrução do olho é impossível; tumores oculares; ou após procedimentos cirúrgicos que cursam com infecção intraocular disseminada (endoftalmite) $)^{(1)}$.

Embora sejam conhecidas as causas de remoção do olho ou de seu conteúdo em alguns serviços de referência, principalmente

Submetido para publicação: 5 de Maio de 2012

Aceito para publicação: 21 de Janeiro de 2013

Trabalho realizado no Departamento de Oftalmologia, Otorrinolaringologia e Cirurgia de Cabeça e Pescoço da Faculdade de Medicina de Botucatu, Universidade Estadual Paulista - UNESP - Botucatu (SP), Brasil.

Médica, Departamento de Oftalmologia, Otorrinolaringologia, Cirurgia de Cabeça e Pescoço, Faculdade de Medicina de Botucatu, Universidade Estadual Paulista - UNESP - Botucatu (SP), Brasil.

Acadêmico de Medicina, Faculdade de Medicina de Botucatu, Universidade Estadual Paulista UNESP - Botucatu (SP), Brasil.

UNESP - Botucatu (SP), Brasil.
Professor Titular, Departamento de Bioestatística, Instituto de Biociências, Universidade Estadual

Professor Titular, Departamento de Bioestatis
Paulista - UNESP - Botucatu (SP), Brasil.

${ }^{4}$ Professora Titular, Departamento de Oftalmologia, Otorrinolaringologia, Cirurgia de Cabeça e Pescoço da Faculdade de Medicina de Botucatu, Universidade Estadual Paulista - UNESP - Botucatu (SP), Brasil.

Financiamento: Não houve financiamento para este trabalho.

Divulgação de potenciais conflitos de interesse: R.L.F.de Sousa, Nenhum; A.R.C.Marçon, Nenhum; C.R.Padovani, Nenhum; S.A.Schellini, Nenhum.

Endereço para correspondência: Roberta Lilian Fernandes de Sousa. Rua Marília, 427 - Apto. 3 Botucatu (SP) - 18608-560 - Brasil - E-mail: rlfsousa@yahoo.com.br

Aprovado pelo Comitê de Ética em Pesquisa da Faculdade de Medicina de Botucatu sob o número 4001/2011. 
serviços de Hospitais-Escola(2), a prevalência de casos de cavidade anoftálmica na população geral não é conhecida. A maioria dos trabalhos relata as técnicas utilizadas para a remoção do olho e avalia sua eficiência(3), não especificando o perfil dos pacientes e nem as causas pelas quais houve a necessidade da cirurgia.

Com o objetivo de mostrar a frequência de ocorrência destes casos na população geral, assim como traçar um perfil dos portadores de cavidade anoftálmica em nosso meio, é que foi realizado este trabalho.

\section{MÉTODOS}

Este estudo é parte de uma pesquisa realizada na região centrooeste do Estado de São Paulo, realizada em 12 cidades para as quais o centro de referência em Saúde é a cidade de Botucatu e que teve como intuito conhecer as causas de cegueira na população geral. Trata-se de um estudo de base populacional, transversal, observacional, realizado nos anos de 2006 a 2008. O tamanho amostral foi estimado em 12.000 indivíduos, tendo por base dados históricos de prevalência de cegueira no nosso meio ${ }^{(4)}$.

Os sujeitos foram escolhidos por sorteio, baseando-se, para estabelecimento da amostra, em dados demográficos do Censo de 2005 e nos setores censitários utilizados naquele censo. Foi sorteada a primeira residência do setor censitário e, a partir dela, foi escolhida a quinta residência do lado ímpar da rua e assim sucessivamente.

Utilizou-se uma Unidade Móvel equipada para atendimento oftalmológico para acessar a população, além das dependências de Postos de Saúde localizados nas cidades visitadas. A equipe era composta por membros da Faculdade de Medicina de Botucatu, além de integrantes das Secretarias de Saúde dos Municípios.

O protocolo da pesquisa foi aprovado pelo Comitê de Ética em Pesquisa da Faculdade de Medicina de Botucatu e consistiu de dados demográficos e exame oftalmológico completo (anamnese, antecedentes oculares e sistêmicos, antecedentes familiares, acuidade visual com e sem correção óptica, tonometria, biomicroscopia, fundoscopia e exame refracional). Os dados obtidos nas consultas foram transferidos para tabela Excel.

Foram examinados 11.453 indivíduos, sendo analisados, no presente estudo, os dados dos portadores de cavidade anoftálmica, observando-se variáveis descritivas tais como sexo, idade, causa da perda ocular e cidade de procedência daqueles indivíduos. A análise estatística foi apresentada segundo a frequência de ocorrência da variável em estudo(5).

\section{RESULTADOS}

Foram examinados 11.453 indivíduos das 12 cidades da região centro-oeste paulista. Destes, 4.586 eram do sexo masculino e 6.867, do sexo feminino (Tabela 1). A tabela 1 mostra ainda, os porcentuais de participantes atendidos em cada Município, em comparação à população total dos mesmos.

Dentre todos os examinados, foram encontrados 11 portadores de cavidade anoftálmica, ou seja, a frequência de ocorrência de cavidade anoftálmica na região estudada foi de 0,96\% (IC 95\% $[0,39 \%$ o $\leq \mathrm{n} \leq 1,53 \% 0]$ ).

Considerando-se o total de participantes da amostra de acordo com o sexo, em valores absolutos, foram examinadas 6.867 mulheres, das quais cinco apresentavam anoftalmia, com frequência de ocorrência de $0,7 \%$, enquanto os homens, para um total de 4.586 homens examinados, foram encontrados seis portadores de cavidade anoftálmica, com frequência de ocorrência de 1,3\%. Ou seja, a anoftalmia foi mais frequente em homens.

Houve uma grande variação quanto à idade dos portadores de cavidade anoftálmica: três indivíduos apresentavam idade entre 10 e 19 anos, três entre 40 e 49 anos, um entre 50 e 59 anos, três entre 60 e 69 anos e três com idade maior que 70 anos (Tabela 2).

Quanto à cor da pele, três portadores de cavidade anoftálmica eram brancos, um era negro e não havia informação da cor da pele em sete destes pacientes. Dos 11 indivíduos, seis apresentavam cavidade anoftálmica à direita e cinco, à esquerda (Tabela 2).

Em relação às causas da perda ocular, dois casos haviam sido submetidos à remoção do olho após trauma ocular, dois por glaucoma, dois por endoftalmite, dois por uveíte e um por malformação congênita (microftalmia). Não foram obtidos dados sobre a causa da perda ocular em dois participantes (Tabela 2).

Sete indivíduos $(63,6 \%)$ residiam na cidade de Botucatu, um era da cidade de Areiópolis, um de Taguaí e dois, da cidade de Piraju.

Dois indivíduos eram aposentados e dois trabalhavam no domicílio. Não foi possível obter dados de profissão de sete portadores de cavidade anoftálmica.

Quanto à acuidade visual do olho contralateral, avaliada pela tabela de Snellen e com o uso da melhor correção óptica, sete apre-

Tabela 1. Distribuição dos casos de cavidade anoftálmica detectados segundo sexo dos participantes do estudo e município de moradia

\begin{tabular}{|c|c|c|c|c|c|}
\hline \multirow[b]{2}{*}{ Município } & \multicolumn{2}{|c|}{ Sexo } & \multirow{2}{*}{$\begin{array}{c}\text { Total de participantes } \\
\text { de cada município }\end{array}$} & \multirow{2}{*}{$\begin{array}{c}\text { Porcentual da população } \\
\text { atendida (\%) }\end{array}$} & \multirow{2}{*}{$\begin{array}{c}\text { Casos de cavidade } \\
\text { anoftálmica }\end{array}$} \\
\hline & Masculino & Feminino & & & \\
\hline Arandu & 278 & 468 & 746 & 12,40 & 0 \\
\hline Areiópolis & 301 & 457 & 758 & 7,13 & 1 \\
\hline Bofete & 269 & 423 & 692 & 8,07 & 0 \\
\hline Botucatu & 1.096 & 1.458 & 2.554 & 2,11 & 7 \\
\hline Conchas & 373 & 640 & 1.013 & 6,54 & 0 \\
\hline Itaí & 347 & 673 & 1.020 & 4,50 & 0 \\
\hline Manduri & 386 & 634 & 1.020 & 11,79 & 0 \\
\hline Pereiras & 352 & 543 & 895 & 12,18 & 0 \\
\hline Pratânia & 256 & 441 & 697 & 16,17 & 0 \\
\hline Piraju & 359 & 441 & 800 & 2,83 & 2 \\
\hline São Manuel & 278 & 167 & 445 & 1,17 & 0 \\
\hline Taguaí & 291 & 522 & 813 & 8,45 & 1 \\
\hline Total & 4.586 & 6.867 & 11.453 & - & 11 \\
\hline
\end{tabular}


Tabela 2. Dados descritivos referentes aos portadores de cavidade anoftálmica detectados na região centro-oeste do Estado de São Paulo

\begin{tabular}{|c|c|c|c|c|c|c|}
\hline Indivíduo & Município de origem & Faixa etária & Cor da pele & Sexo & Olho afetado & Causa da perda do olho \\
\hline 1 & Areiópolis & $60-69$ & Negra & Masc & OD & Trauma \\
\hline 2 & Botucatu & $\geq 70$ & - & Fem & OD & Trauma \\
\hline 3 & Botucatu & $60-69$ & - & Masc & OD & Endoftalmite \\
\hline 4 & Botucatu & $50-59$ & - & Masc & $\mathrm{OE}$ & Sem dados \\
\hline 5 & Botucatu & $40-49$ & - & Fem & OE & Sem dados \\
\hline 6 & Botucatu & $10-19$ & - & Masc & $\mathrm{OE}$ & Sem dados \\
\hline 7 & Botucatu & $\geq 70$ & - & Fem & $\mathrm{OD}$ & Sem dados \\
\hline 8 & Botucatu & $60-69$ & - & Masc & OD & Endoftalmite \\
\hline 9 & Piraju & $40-49$ & Branca & Fem & $\mathrm{OE}$ & Uveíte \\
\hline 10 & Piraju & $40-49$ & Branca & Fem & OE & Glaucoma \\
\hline 11 & Taguaí & $\geq 70$ & Branca & Masc & OD & Glaucoma \\
\hline
\end{tabular}

Fem= feminino; Masc $=$ masculino; $\mathrm{OD}=\mathrm{olho}$ direito; $\mathrm{OE}=$ olho esquerdo

sentavam visão maior ou igual a 0,7, um apresentava visão entre 0,3 e 0,7 e um tinha visão menor que 0,05. Um dos indivíduos em que a perda ocular ocorreu por glaucoma, apresentava buraco macular no olho contralateral.

Dois dos portadores de cavidade anoftálmica eram diabéticos e os demais não apresentavam alterações sistêmicas relacionadas com a causa da perda ocular.

Dez pacientes receberam prescrição de novas lentes para o olho contralateral e apenas uma correção óptica foi mantida.

\section{DISCUSSÃO}

Estudos sobre cavidade anoftálmica e causas de perda do bulbo ocular são escassos, podendo-se dizer que este é o primeiro estudo de base populacional e aleatorizado envolvendo portadores de cavidade anoftálmica.

O presente estudo foi desenvolvido utilizando dados de estudo mãe sobre as causas de cegueira na população de determinada região do Estado de São Paulo onde a atenção à Saúde é feita pela Faculdade de Medicina de Botucatu(4). Para aquele estudo, o $n$ amostral foi baseado em estudos prévios que apontavam para uma taxa de cegueira na população de cerca de 1\%. Assim, foi determinado que cerca de 12 mil pessoas deveriam ser examinadas, em uma população escolhida ao acaso. A oportunidade de se encontrar portadores de cavidade anoftálmica, ou seja, a frequência de ocorrência de cavidade anoftálmica, em habitantes desta região foi de 0,96\%. 0 total de pacientes atendidos equivale a uma média de $7,77 \%$ do total de habitantes destas cidades, porcentual significativo e que pode realmente expressar as características da comunidade estudada ${ }^{(6)}$.

Embora a frequência de portadores de anoftalmia seja baixa, a perda do olho ou de seu conteúdo deixa sequela e incapacidade importante e é sempre o intuito do oftalmologista evitá-la.

Considerando-se o total de participantes da amostra de acordo com o sexo, a frequência de ocorrência de cavidade anoftálmica em mulheres foi de 0,7\%, enquanto nos homens foi de 1,3\%o, quase o dobro do que apresentaram as mulheres. É clássico considerar os homens mais sujeitos a traumas, tanto recreacionais, como decorrentes de atividades laborais ${ }^{(2)}$, com maiores chances de perder o olho que as mulheres.

Com relação à idade no momento da coleta dos dados, não houve predominância de ocorrência da cavidade anoftálmica em uma determinada faixa etária. No entanto, a idade da perda não foi apurada. Com esta ressalva, nota-se que a maioria dos indivíduos apresentava idade acima de 60 anos na época do levantamento.
Quanto à cor da pele, a informação estava ausente em porcentual expressivo dos portadores, o que dificulta a avaliação do parâmetro. Entretanto, não há motivo para considerar que a cor da pele possa influenciar na perda do bulbo ocular.

Não houve influência da lateralidade na perda do bulbo.

Nossos dados confirmam que, em que pese o desenvolvimento de novos tratamentos ou técnicas operatórias, o trauma, as infecções (endógenas ou exógenas) e o glaucoma, continuam sendo importantes causadores de perdas irreversíveis para os olhos ${ }^{(7)}$.

Há estudos que apontam os problemas acontecidos na zona rural como potencialmente piores para o olho, devido à dificuldade de se ter atendimento especializado em curto espaço de tempo. O presente estudo levantou apenas a condição presente na área urbana, não sendo possível comparar com a zona rural.

A avaliação do olho contralateral permitiu reconhecer condição de cegueira segundo a definição da $\mathrm{OMS}^{(7)}$ que seria acuidade visual pior que 0,05 em ambos os olhos em apenas uma pessoa. Nas demais, o olho contralateral encontrava-se com visão que poderia ser considerada normal. Ressalte-se que houve necessidade de nova correção óptica em 10 dos 11 indivíduos, ou seja, apenas o que se encontrava em condição de cegueira não se beneficiou com mudança de lentes corretivas. Outro benefício das lentes corretivas seria a proteção do olho contralateral de possíveis traumas, o que diminuiria a chance de cegueira bilateral|(8).

Importante também observar que um dos pacientes que havia perdido um olho por glaucoma, apresentava outra alteração no olho contralateral que impedia a boa visão, o que mostra a importância de exame completo na determinação das reais causas de perda de visão.

Levando-se em conta as causas de cegueira na população, na região do estudo, a prevalência de cegueira é de $0,4 \%{ }^{(4)}$ e de portadores de cavidade anoftálmica é de 0,96\%o. Perder a visão é considerado desastroso. No entanto, a remoção do olho ou de seu conteúdo, mesmo que a visão esteja ausente, é buscada somente em casos extremos, em condições de dor insuportável, quando há tumores extensos ou prejuízo estético muito importante. Nos demais casos, muitas vezes até por se ter esperança ainda de se obter qualquer melhora ou até mesmo por tratar-se de procedimento mutilante, o indivíduo protela a realização destes procedimentos. Talvez esta seja a razão da diferença tão marcante entre o número de pessoas cegas e o número de portadores de cavidade anoftálmica.

O oftalmologista precisa conhecer o portador de cavidade anoftálmica, observando seu perfil e suas ansiedades. Conhecer o perfil e as causas desta condição permite instalar serviços de prevenção. Conhecer a prevalência destes casos, por sua vez, é uma excelente 
forma de melhorar o atendimento aos pacientes, permitindo a implantação de serviços que possam ajudá-los a enfrentar a nova realidade de portadores de cavidade anoftálmica.

\section{CONCLUSÃO}

A frequência de ocorrência de cavidade anoftálmica em habitantes da região centro-oeste paulista foi de 0,96\%o, acometendo principalmente homens e com grande variação de idade de acometimento.

\section{REFERÊNCIAS}

1. Burgett RA, Nunery WR. Órbita anoftálmica. In: Chen WP. Cirurgia plástica oftalmológica: princípios e prática. Rio de Janeiro: Revinter; 2005. Cap 26, p.369-85.
2. Mattos BS, Carvalho JC. Prevalência das perdas do globo ocular. I. Estudo das variáveis lado, etiologia, sexo. Rev Odontol Univ São Paulo. 1988;2(3):175-81.

3. Su GW, Yen MT. Current trends in managing the anophthalmic socket after primary enucleation and evisceration. Ophthal Plast Reconstr Surg. 2004;20(4):274-80.

4. Schellini SA, Durkin SR, Hoyama E, Hirai F, Cordeiro R, Casson RJ, et al. Prevalence of refractive errors in a Brazilian population: the Botucatu eye study. Ophthalmic Epidemiol. 2009;16(2):90-7.

5. Norman GR, Streiner DL. Biostatistics: the bare essentials. 3rd ed. USA: People's Medical Publishing House; 2008. p. 393.

6. Instituto Brasileiro de Geografia e Estatística - IBGE. Disponível em: HTTP://www.ibge. gov.br

7. ResnikoffS, Pascolini D, Etya'ale D, Kocur I, Pararajasegaram R, Pokharel GP, et al. Global data on visual impairment in the year 2002. Bull World Health Organ. 2004;82(11): 844-51.

8. Bilyk JR. Enucleation, evisceration, and sympathetic ophthalmia. Curr Opin Ophthalmol. 2000;11(5):372-86

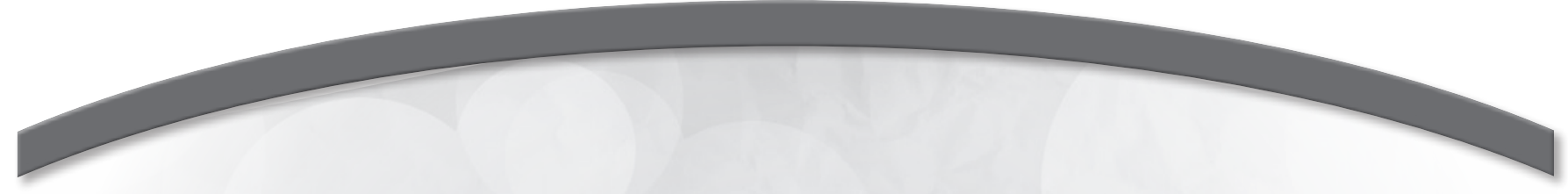

\section{Encontro Anual da} Academia Americana de Oftalmologia

\section{6 a 19 de novembro de 2013}

Nova Orleans, Louisiana (EUA)

Informações:

Site: www.aao.org

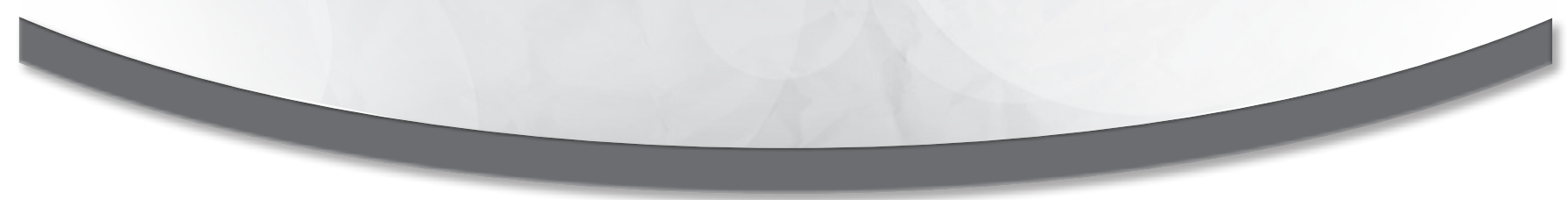

